\title{
INCURSIÓN DE LOS Y LAS JÓVENES EN EL MUNDO UNIVERSITARIO: TENSIONES ENTRE SER Y PERMANECER
}

\author{
OFELIA ROLDÁN VARGAS*
}

\begin{abstract}
El ser humano no es sólo sus determinaciones sino también sus posibilidades.
\end{abstract}

(Joan Carles Mèlich, 2002)

\begin{abstract}
RESUMEN: Este artículo presenta los resultados del componente Jóvenes recién llegados a la Universidad del estudio macro Configuración del sujeto niño-Joven en la cotidianidad escolar, realizado en el Curso Posdoctoral en Investigación en Ciencias Sociales, Niñez y Juventud, ofrecido por instituciones latinoamericanas. Sustentado en fundamentos hermenéuticos y mediante grupos focales y entrevistas abiertas a 12 jóvenes de Medellín, el estudio permitió analizar la categoría Experiencias del primer año de formación universitaria, cuyos resultados se agrupan en tres tendencias: incursión en el ambiente universitario instituido: entre la experiencia y la acomodación; el devenir sujeto en el contexto de la universidad y el sujeto estudiante como vértice del mundo universitario.
\end{abstract}

Palabras clave: Educación superior. Experiencia. Jóvenes universitarios. Subjetividad.

YOUNG PEOPLE ENTERING THE UNIVERSITY WORLD: TENSIONS BETWEEN BEING AND REMAINING

ABSTRACT: This article presents the results of the investigation Young people as University newcomers which is part of the comprehensive study Configuring the subject child-young person in the University everyday life, carried out in the Postdoctoral course in Investigation in Social Sciences, Childhood and Youth, offered by Latin American institutes. Sustained in hermeneutic foundations and by means of focal groups and open interviews with 12 young individuals from Medellin, the study enabled the analysis of the category Experiences in the first year of University, whose results are grouped under three tendencies: entering the institutionalized University environment:

* Fundación Centro Internacional de Educación y Desarrollo Humano (Cinde). Colombia. Contato com a autora:<oroldan@cinde.org.co> 
from the experience to fitting in; becoming a subject in the university context and the subject student as a vertex of the university world.

Key words: Higher education. Experience. Young college students. Subjectivity.

\title{
INCURSION DES JEUNES DANS LE MONDE UNIVERSITAIRE: TENSIONS ENTRE ÊTRE ET RESTER
}

\begin{abstract}
RÉSUMÉ: Cet article présente les résultats de la composante Nouveaux étudiants à l'université de l'étude Configuration du sujet enfant-jeune dans le quotidien scolaire, réalisée au long du cursus post-doctoral en Recherches de Sciences Sociales, de l'Enfance et de la Jeunesse, offert par des institutions latino-américaines. Soutenue sur des fondements herméneutiques et devant les groupes de discussions et des entretiens ouverts avec 12 jeunes de Medellín, l'étude a permis d'analyser la catégorie Expériences de la première année de formation universitaire, dont les résultats se regroupent en trois tendances: incursion dans l'environnement universitaire, entre l'expérience et l'accomodation; l'acte de devenir sujet dans le contexte de l'université et le sujet étudiant comme le point le plus considérable du monde universitaire.
\end{abstract}

Mots-clés: Éducation supérieure. Expérience. Jeunes universitaires. Subjectivité.

\section{Introducción}

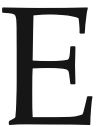
ste artículo da cuenta de los resultados del componente Jóvenes recién llegados a la universidad de la investigación macro denominada Configuración del sujeto niño-joven en la cotidianidad escolar, realizada en el contexto del Curso Posdoctoral en Investigación en Ciencias Sociales, Niñez y Juventud del convenio Universidad de Manizales, Fundación Centro Internacional de Educación y Desarrollo Humano (Cinde, Colombia) y la Pontifícia Universidad Católica de São Paulo (PUC-SP, Brasil) con el aval de Consejo Latinoamericano de Ciencias Sociales (Clacso, Argentina), en la que participaron investigadores/as de la Universidad de la Patagonia (Chile), Universidad de la Sabana (Colombia) y Cinde (Colombia). El estudio se inscribe en el grupo de investigación Educación y Pedagogía: saberes, imaginarios e intersubjetividades de Cinde y la Universidad de Manizales.

La revisión de estudios de carácter local, nacional e internacional sobre el primer año de formación universitaria devela la inclinación de éstos a identificar la capacidad adaptativa de los estudiantes, el efecto de las estrategias institucionales para favorecer su retención y las causas, en cifras, que finalmente provocan la deserción; destacándose entre ellas la incapacidad de permanecer en un contexto educativo exigente académicamente, que resulta ajeno y en muchas ocasiones hostil. Frente al vacío que se nota en los estudios previos respecto de la indagación por aspectos más cercanos a la condición subjetiva del estudiante surge el componente investigativo Jóvenes recién llegados a la universidad, cuyo propósito radica en el acercamiento 
comprensivo a su experiencia de un año, ya no en cifras, sino en condiciones para expandir su libertad, reconocerse en la diversidad y configurarse en espacios de interacción.

De acuerdo a los resultados del estudio, el texto incluye tres aspectos que, de manera complementaria, dan cuenta de la experiencia de incursión de los jóvenes en el mundo universitario; por un lado, se analizan los dos caminos que emergen a la llegada: vivir la experiencia o acomodarse a lo establecido en un contexto universitario que tiene años e incluso siglos de existencia; por otro lado, se desarrolla el interés de los estudiantes por el devenir sujeto en el contexto universitario y, finalmente, se discute el posicionamiento del sujeto estudiante como vértice del mundo universitaria.

Con base en los anteriores resultados, es posible afirmar que la experiencia iniciática de formación universitaria es absolutamente trascendental en la vida de los estudiantes porque no sólo les permite construir sentidos, acerca de la educación y el impacto de ésta en su configuración como sujetos, sino que también puede ayudar a perpetuar o a transformar la institución educativa, en su doble función de producir conocimiento y trascender la cultura.

\section{Método}

Éste es un estudio cualitativo, de corte hermenéutico, soportado en la interpretación de textos producidos social y culturalmente por estudiantes, que busca comprender los sentidos generados en el escenario universitario, en este caso, caracterizado por la incertidumbre de la llegada, la búsqueda de la permanencia y la esperanza del éxito. Se opta por la comprensión, concebida como reconstrucción de una estructura de sentido, a través de espacios de diálogo, por la posibilidad que ofrece de "salir de sí mismo, pensar con el otro y volver sobre sí mismo como otro" (GADAMER, 2005, p. 356).

En el proceso investigativo participaron 12 estudiantes universitarios, hombres y mujeres, con edades entre los 17 y los 25 años, pertenecientes a diferentes estratos socioeconómicos, que cursaban su primer año de educación superior en cuatro universidades de Medellín, dos públicas y dos privadas. La participación fue voluntaria como respuesta a una invitación directa que se hizo en 8 grupos de estudiantes que cumplían con los criterios de inclusión antes descritos, la cual se ratificó con la firma del consentimiento informado. La decisión de incluir sólo estudiantes de primer año está soportada en la necesidad de un acercamiento comprensivo a la situación vivida en esa etapa inicial de estudios superiores, bastante marcada en el contexto por problemas de deserción, repitencia y desadaptación. 
Los datos fueron construidos mediante relatos de experiencias en el contexto de dos grupos focales de 6 estudiantes cada uno, desarrollados por fuera del ambiente universitario y con una duración de dos y media horas. En el primer grupo, de las universidades privadas, participaron 4 estudiantes de los programas de: Ingeniería de Sistemas, Psicología, Fisioterapia y Derecho de estratos 4, 5 y 6, y dos de estratos 1 y 2 , de Medicina y Contaduría Pública, becados por el Programa de Cobertura Educativa. El segundo grupo, de universidades públicas, estuvo constituido por estudiantes de Ingeniería Civil, Licenciatura en Educación Infantil, Administración de Empresas, Medicina, Contaduría y Odontología, distribuidos en estratos 1, 2 y 3, algunos de ellos procedentes del sector rural. Así mismo, se realizaron entrevistas abiertas en el espacio de la cotidianidad universitaria a 8 de estos estudiantes con el propósito de ampliar los argumentos presentados en los grupos focales y acceder a otros tópicos de interés para el estudio.

El análisis se hizo sobre la base de la codificación de la información, entendida por Coffey y Atkinson (1996, p. 33) como una forma de "condensar el grueso de los datos en unidades analizables, creando categorías con ellos o a partir de ellos, para abrir la indagación y pasar a la interpretación". Mediante sistematización de la información y codificación abierta se identificaron regularidades; se develaron las categorías emergentes y sus respectivas tendencias, mediante codificación axial y, finalmente, se hizo la construcción de sentido o desarrollo de las categorías y las tendencias que las constituyen, produciendo así un texto en el que se muestra a manera de entramado de relaciones las experiencias de los jóvenes comenzando su formación universitaria.

\section{Aproximación comprensiva a la experiencia universitaria iniciática}

\section{Incursión en el ambiente universitario instituido: entre la experiencia y la acomodación}

Es incapaz de experiencia el que se pone, o se opone, o se impone, pero no se ex-pone. (LARROSA, 2009)

Como consecuencia de toda situación signada por la incertidumbre del comienzo, quienes tienen el privilegio de llegar a la universidad - en su primer año de estudio - incursionan en un ritual de paso, que aunque temporal, marca de manera significativa sus vidas en tanto puede reforzar en ellos su condición de sujetos ajustados o acomodados que encuentran en la acumulación de información, en la apropiación de elementos técnico-científicos y en la capacitación para el hacer y responder a las demandas de un mundo productivo que no cesa de moverse, el único o más preciado sentido de su proceso formativo; pero también está latente la posibilidad de que este 
primer contacto con el ethos universitario los convierta en sujetos de la experiencia, es decir, sujetos que viven una transformación positiva en sus modos particulares de ser, pensar, actuar y relacionarse.

El primero y tal vez el más grande reto que tienen que enfrentar los y las jóvenes recién llegados a la universidad es armonizar la vivencia de la discontinuidad de un proceso educativo transicional lleno de incertidumbres con la continuidad de su vida y de su aprendizaje. Mientras sus condiciones cotidianas, muy probablemente, cambian de manera radical y los ubican al vaivén de los requerimientos de un mundo educativo desconocido, sus ideas, sus deseos, sus sueños y su pasión por lo quieren ser y hacer son tocados de una manera más lenta, dependiendo claro está de la conciencia con que hayan sido construidos y de la fuerza con la que se intencione el impacto del proceso educativo que inician.

De acuerdo a lo anterior, el momento de la transición cobra gran importancia porque allí está el sujeto, configurado hasta entonces en otras experiencias que marcan el antes de la universidad, y frente a él las nuevas posibilidades de seguir siendo o llegar a ser de otra manera en el contexto universitario, que representan el después con todas sus implicaciones. Surge aquí el punto crucial que devela la problemática a la que se enfrenta el sujeto, que recientemente se convierte en estudiante universitario, por el requerimiento que se le hace de ajustarse a unas condiciones e interiorizar unos valores institucionales que se imponen desde el momento de la inducción y se refuerzan en los procesos posteriores de socialización, tal como lo confirma este texto: "sólo hay una alternativa que es clara desde la inducción, uno hace lo que le dicen y se adapta rápidamente o corre el riesgo de salir en el intento y ahí sí, se perdió todo" (Camilo, 18 años ) .

Aunque Martuccelli (2007, p. 44) plantea que "la interiorización de las normas y la encarnación del rol social devienen menos importantes a la hora de caracterizar al actor, que la gestión de la distanciación del individuo hacia sus propias imágenes sociales", en este caso particular de vinculación al mundo universitario en países donde la educación superior, más que un derecho de toda la población, es un privilegio de unos pocos, sucede todo lo contrario. Es tan fuerte el temor de perder el cupo que, de manera consciente, se aplazan apuestas vitales y entre tanto se le da mayor peso a la interiorización normativa de lo social y culturalmente reconocido dentro del modelo institucional, lo mismo que a la incorporación de las disposiciones que se han ido configurando como política universitaria.

Situaciones como la presión de pocos cupos para muchos aspirantes en instituciones oficiales, como lo evidencia el bajo porcentaje de aspirantes admitidos en el primer semestre de 2013 en las dos principales instituciones públicas de educación superior de Medellín: el 16.8\% en la Universidad de Antioquia y el 15.6\% en la Universidad Nacional de Colombia, o el pago de costos altos por concepto de 
matrículas en establecimientos privados llevan a que la adaptación o acomodación acrítica al ethos universitario aparezca con frecuencia como apuesta primera en el horizonte cotidiano del estudiante que inicia, negándose de esta manera la posibilidad de tener una verdadera experiencia, entendiendo por ello el espacio abierto en el que cada uno puede configurar su diferencia, como lo manifiestan los estudiantes: "yo no puedo ser libre como quisiera tengo que esperar a salir de la universidad" (Felipe, 17 años) ), "no sería justo arriesgar tantos millones que paga mi papá por ponerme de brabucón o querer hacer lo que me gusta " (Santiago, 16 años), "entrar a la U no es tan fácil por eso tengo que abandonar muchas cosas de mi vida para rendir y no dejarme sacar" (Tatiana, 18 años), "mi familia tiene una empresa y mi papá me insistió que hiciera esta carrera para que lo apoyara y también porque se con esto puedo tener una vida económica tranquila"(Mauricio, 17 años). La presión de permanecer en la universidad y de aprobar los semestres con miras a la titulación en el tiempo previsto es tan alta que se convierte en una idea fija o en meta única que aplaza el disfrute del aprendizaje y el desarrollo de una conciencia crítica frente a las situaciones cotidianas, por razones que van desde el afán de concluir para vincularse al mundo laboral y pagar la deuda adquirida por concepto de matrícula o por la presión de responder a encargos familiares de perpetuar tradiciones profesionales y deseos personales de garantizar/se la pertenencia a buen estatus socioeconómico.

Si la formación es experiencia y "la experiencia es siempre de alguien, subjetiva, es siempre de aquí y de ahora, contextual, finita, provisional, sensible, mortal, de carne y hueso, como la vida misma (LARROSA, 2004, p. 167), las voces de los participantes develan un vacío importante en su proceso formativo, en tanto, muestran poco interés de estos recién llegados por ocuparse expresamente de tramitar la disyuntiva entre romper con un ethos instituido que se impone y poner en riesgo su permanencia; entre los apegos a lo conocido, por la seguridad que representa, y la apuesta por lo desconocido, en tanto potencial movilizador de nuevos desarrollos; entre la nostalgia por ciertas relaciones anteriores que tendrán que modificarse e incluso quedar suspendidas en el tiempo, y las expectativas frente a nuevos vínculos relacionales que empiezan a configurarse; entre la emoción asociada al desequilibrio por nuevas formas de pensamiento y la aparente seguridad que da la acomodación cognitiva.

En este sentido, sorprende que los estudiantes universitarios recién llegados sólo hagan mención pasajera a aspectos tan vitales como los anteriormente anotados y que en cambio si les preocupe demasiado cómo estirar el tiempo para responder, en muchos casos de manera acrítica, a las múltiples responsabilidades académicas que les son asignadas; cómo estar activos en las prolongadas y extenuantes jornadas escolares, cómo idearse la forma de silenciar los reclamos amorosos de quienes les extrañan en los espacios y horarios cotidianos, cómo olvidar el dolor que provoca el cambio obligado de actividades placenteras por ejercicios académicos impuestos 
y que mientras todo esto sucede poco o nada realmente trascendental pase en ellos, asociando lo trascendental con experiencia y entendiendo por experiencia todo aquello que moviliza el pensamiento y la acción, que convoca a la renovación, que estimula lo esencialmente humano.

Expresiones como: "en la semana no puedo jugar con mi hija de dos años porque salgo en la madrugada y cuando llego está dormida" (Claudia, 20 años); "he tenido muchos problemas con mi pareja porque siempre estoy estudiando porque no me puedo dejar sacar de U. que se me volvió la única meta ahora" (Diego, 19 años); "Mi gran pasión era el deporte pero eso se acabó mientras estudio medicina, ufff si uno se descuida sale, en la U. es muy duro" (Mauricio, 17 años), "... sin bajar la calidad, yo pienso que el estudio universitario podría ser más humano, pero cuando uno hace algún reclamo los profes responden que aquí se vino fue a estudiar, muchas veces me siento sin fuerzas y aislada de mi familia y eso que apenas estoy empezando" (Carolina, 18 años) evidencian una sujeción fuerte por parte de los y las jóvenes a los requerimientos del mundo académico, un mundo que se construye desde la perspectiva de sujetos generalizados y homogenizables en cuanto a los resultados que de ellos se esperan, en el que no se alcanza a "ver a cada ser racional como un individuo con historia, identidad y constitución afectivo-emocional concreta" (BENHABIB, 2006, p. 183).

\section{El devenir sujeto en el contexto universitario}

El sabor de la manzana no está ni en la boca que la muerde ni en la carnosidad de la manzana, sino en el encuentro entre ambas. (BORGES citado por BÁRCENA, 2002)

A problemáticas como el recorte financiero en todos los ámbitos, la recesión económica que alerta a los grandes inversionistas de la educación incluido el Estado, la promesa no cumplida de una educación de calidad para todos que ayude a cerrar la brecha entre los pocos que tienen mucho y los muchos que tienen poco, y el retraimiento del mercado laboral, que hace cada vez más esquiva la posibilidad de empleos dignos garantes de una vida igualmente digna para el grueso de la población, se suma con fuerza el estímulo interno y también estatal para las instituciones con alto desempeño académico y menores índices de repitencia y deserción, lo cual no sólo aviva la expectativa de competencia interinstitucional en el medio social sino que también da origen al "profesor retencionista, que considera su deber ayudar al estudiante a ser tan exitoso como sea posible" (SIMPSON, 2010, p. 19), en términos de rendimiento académico.

La anterior apuesta, apoyada en la concepción de éxito educativo como retención y culminación de una carrera profesional con buenas calificaciones, deja entrever 
empobrecimiento de la misión humana, educativa y social de la universidad y reduccionismo significativo de la función docente, tal como lo expresan los estudiantes: "Los profesores se obsesionan tanto por el rendimiento académico que uno llega a creer que es lo único que les importa" (Camilo, 18 años), "así como cuidan que los estudiantes no se vayan debería haber más cuidado para revisar las condiciones en que están los que se han quedado, digo, en lo que está pasando internamente en sus vidas" (Tatiana, 18 años).

En situaciones irregulares y precarias, en las que son pocos los botines y muchos los exploradores, hay un peligro latente de dejarse entrampar por la codicia del número, reduciendo el éxito educativo a indicadores como la retención hasta la titulación, los marcadores altos en aprobación semestral, las mejores calificaciones en pruebas estandarizadas y la competición por puntear el más alto índice de rendimiento académico, desconociendo la complejidad de los procesos educativos y dejando de lado las posibilidades fascinantes que subyacen en la educación para el desarrollo humano, entendido éste como la constitución del sujeto, con conciencia de sí y del mundo, capaz de vincularse y crear condiciones para que otros y otras también se configuren como sujetos posicionados histórica, social, económica, cultural y políticamente, es decir, para que unos y otros se constituyan en proyecto individual y colectivo.

De acuerdo a lo anterior, la función de la educación no puede ser otra que el cultivo del potencial de humanidad que hay en cada ser - entiéndase por ello la provocación del desarrollo de sus dimensiones físico madurativa, laboral, cognitiva, afectivo erótica, lúdico estética, espiritual, ético moral y política - lo cual implica un ejercicio simultáneo y en dos vías. Por un lado, auto-reconocimiento por parte del sujeto de lo que es y tiene, de lo que desea ser y tener, y de su disposición para expandir la imaginación y dar cabida a lo que no conoce de sí mismo y del mundo, a lo imprevisible, a lo porvenir; y por otro lado, reconocimiento del sujeto en su particularidad por parte de la institución y de quienes orientan los procesos educativos para evitar la fragmentación de ese ser, que es integral, en parcelas de intervención o su conversión en objeto de homogenización, como lo advierten los estudiantes: "me preocupa que a los 'nerd' se les ensalce tanto sin analizar sus relaciones con los demás y su solidaridad porque algunos se creen dioses y usted se imagina cómo van a ser cuando sean profesionales?" (Carolina, 18 años), "Uno como universitario tiene que saber qué le gusta y qué no, cómo quiere ser, y el estudio debería servir para eso" (Felipe, 16 años).

En este mundo contemporáneo, en apariencia diverso pero en el fondo bastante homogenizado por ciertas metas globales, por la producción en serie, la concentración del poder y la mercantilización de lo humano en el que la cotidianidad se repite demasiado, las rutinas se instalan con gran facilidad y las realidades se estrechan de 
tal modo que parecieran agobiar la imaginación, la educación tiene el reto de convertirse en acontecimiento, es decir, en "un estallido de sentido, algo no programable, una irrupción imprevista: el comienzo de una nueva narrativa, de una nueva comprensión" (BÁRCENA, 2004, p. 76) que le ayude al sujeto a encontrar lugar para expandir/se y darse otra forma "más humana, menos dependiente de la apariencia, de la competencia y el acaparamiento que tanto carcome el alma y genera violencia" (Claudia, 20 años).

Es precisamente la educación, entendida como acontecimiento generador de experiencias transformadoras, la vía más expedita hacia

[...] procesos de apropiación del sentido que viene del mundo al sujeto, que el sujeto evalúa en su confrontación con las expresiones de otros y con el darse de la experiencia cotidiana hasta llegar a ser $s u$ sentido - voz propia - que significa de nuevo sentido del mundo de la vida. (VARGAS, 2007, p. 25)

En esta referencia a la educación como acontecimiento generador de experiencias transformadoras, hay una apuesta clara por la educación como natalidad, suceso u ocurrencia de algo nuevo, que sin tener el carácter de extraordinario, es potencialmente capaz de transformar las formas de relacionamiento que alienan y servir de germen para el cultivo permanente de humidad. De manera complementaria hay una apuesta por el sujeto de la experiencia, es decir, por el sujeto que se deja tocar por el conocimiento, bien sea porque lo construye o porque accede a él mediante otras fuentes, y permite que algo suceda en él, en su pensamiento, en su conciencia, en sus prácticas, en su cotidianidad, en sus expectativas de vida y en su manera de habitar el mundo y configurarlo en espacios de intersubjetividad.

Desde esta perspectiva, el sentido, dígase de la vida, de la educación, de la profesión, de lo humano, aunque es particular de cada sujeto, se construye en espacios de relación con otros, en escenarios concretos de actuación y gracias al acontecer cotidiano en el que está implícita la conciencia. Tomar conciencia de alguien, en el caso particular de la educación, es asumirlo en serio, es abrirle un espacio en la propia vida para darle cabida a su densidad existencial, acogerlo como un otro, dispensarle cuidado, ofrecerle posibilidad y ayudarle a devenir sujeto en toda su integralidad, "es enseñarle a ser, es más que ponerle una nota, es mucho más que retener hasta graduar" (Daniela, 19 años), es crear con él otro tiempo para que a su vez cree su propio tiempo, distinto al "tiempo de la educación que es un tiempo cronometrado, un tiempo tan acelerado, tan rápido, que casi no da tiempo para que nada nos pase, porque nada vemos pasar mientras avanzamos en ese paso incesante" (BÁRCENA, 2012, p. 52).

Queda así planteada la acogida y el acompañamiento a los estudiantes en el cultivo de su humanidad como una de las funciones del maestro universitario. 
Su presencia dinamizadora ha de representar apoyo significativo en el proceso de configuración como sujetos, contribuir a que marquen su diferencia y construyan su identidad mientras le imprimen movimiento a un mundo colectivo que ya se movía a la llegada de cada uno, a la manera de gran obra de teatro, cuyo guión no tiene ni principio ni fin pero sí espacio para el encuentro polifónico de voces que se vuelven sobre sí mismas para autodefinirse y autodeterminarse, sin perder la secuencia de la trama plural ni dejar escapar el suspenso asociado a cada desenlace. Así entendidos la acogida y el acompañamiento, resultaría demasiado reduccionista centrar la atención en mantener la correspondencia numérica entre matriculados y titulados, entre ingresos y permanencias, perdiendo quizás de vista las escenas intermedias y su potente contribución a la expresión del sí mismo y a la configuración del nosotros, entendidos como finalidades importantes de la educación.

El equipo docente que acompaña el proceso universitario iniciático tiene una función doble de gran trascendencia. Por un lado, es suya la responsabilidad de facilitar el acercamiento comprensivo al ethos universitario, ayudando a descubrir el potencial que subyace en él para la formación profesional, entendiendo que de ello también hace parte la configuración subjetiva, $\mathrm{y}$, simultáneamente, tiene el compromiso ético de proporcionar condiciones para la lectura crítica de la cotidianidad universitaria que le permita a los estudiantes tomar decisiones suficientemente fundamentadas entre apoyar continuidades o provocar rupturas. De este modo, además de preocuparse por la permanencia y garantizar los aprendizajes requeridos para un desempeño laboral de calidad consciente, crítico y transformador, el equipo docente tendría que destacarse por el "acogimiento hospitalario de los recién llegados, por una práctica ética interesada en la formación de la identidad de los sujetos" (BÁRCENA; MÈLICH, 2000, p. 15).

Centrar el interés de la educación en volver competentes a los estudiantes para el desempeño de una profesión responde al paradigma de la "Pedagogía de la programación, de la evaluación, de la previsibilidad. Una pedagogía del poder. Una pedagogía tecnológica en definitiva" (ibid., p. 43), en la que se desdibujan los rostros, el propio y los de los demás, en tanto las relaciones se establecen entre roles y a partir de las funciones que programáticamente se les asignan a cada uno de ellos. Queda, entonces, la gran apuesta por una educación con rostro en la que sea posible ver al ser humano que habita en el estudiante y en el trabajador, y a la dimensión laboral sólo como una entre muchas otras a desarrollar. Una educación, en la que "la palabra de un otro nos trasciende como educadores frente al cual tenemos la obligación de asumir una responsabilidad incondicional más allá de todo contrato posible o de reciprocidad" (ibid., p. 35).

Atreverse a ver en los que apenas llegan al mundo universitario seres con multivariados acervos de conocimiento, con interrogantes resueltos y muchos otros 
apenas en estado incipiente de formulación, con grandes habilidades comunicativas y para el manejo tecnológico propias de su generación, en otras palabras, con mucho que aportar y contradecir, implica hacer una revisión crítica bastante juiciosa del ethos universitario, organizado a lo largo de la historia desde la lógica de los grandes procesos estructurales regidos por las nociones de disciplinamiento, adaptación, homogeneidad, transmisión y estándares.

Provocar el descubrimiento del ser concreto que hay en cada estudiante requiere un acercamiento mucho más comprensivo a la cotidianeidad universitaria, tratando de descubrir en ella al sujeto de la enteridad planteado por Maffessoli (2004), es decir, al sujeto concebido en su incompletud y complejidad; al sujeto concreto poseedor de certezas temporales y también depositario de incertidumbres movilizadoras; al sujeto con "capacidad de pensar por sí mismo, sin desconocer al otro, y reconocer, crear, disponer en la práctica los principios que orientan la vida" (CUBIDES, 2004, p. 124), al sujeto que no sólo le da sentido a las estructuras sino que se convierte en el motivo y la razón primera de la acción educativa.

Entender así la misión de ser docente, en este momento fundamental de la iniciación universitaria, es comprender con Arendt (citada por Bárcena y Mèlich, 2000, p. 89), que "la educación es el punto en que amamos al mundo lo bastante como para asumir una responsabilidad por él y así salvarlo de la ruina que de no ser por la renovación, de no ser por la llegada de los nuevos y los jóvenes sería inevitable".

\section{El sujeto estudiante como vértice del mundo universitario}

La comprensión de los fenómenos sociales contemporáneos exige una inteligencia desde los individuos. (MARTUCCELLI, 2007)

El planteamiento de Martuccelli sobre la importancia de partir del sujeto para comprender los fenómenos sociales, que se recoge en el epígrafe, ilustra la manera como los estudiantes desean que fuese la universidad y su rol particular en ella, es decir, no centrada en ella misma y tampoco como una organización que se estructura y desarrolla al vaivén de las tendencias y los requerimientos del mercado global, sin importar mucho las especificidades de los sujetos que la constituyen y le dan sentido a su acción cotidiana. Desde esta perspectiva, se descentra la universidad como estructura explícitamente organizada en términos de una productividad regida por principios de rendimiento, eficiencia, eficacia y competitividad en el contexto global-local y se posiciona al sujeto estudiante como vértice del mundo universitario, lo cual no significa que se reduzca la dinámica institucional al nivel del sujeto o que se apueste por una educación sin previa lectura de la sociedad en su conjunto. En esta 
apuesta en y por el sujeto, es decir, por su potencial de novedad, su capacidad renovadora o, como diría Arendt, comentada por Bárcena y Mèlich (2000, p. 6), por una filosofía de la natalidad entendida como punto de partida o inicio en el que cada sujeto se exprese en términos de "una radical capacidad de comenzar algo nuevo y sorprendente que no estaba previsto", se conjugan dos propósitos: humanizar la educación, tan impregnada hoy por la lógica del servicio mercancía, y re-fundamentar la universidad, para que pueda promover tal humanización.

Dar este giro trascendental que centra la atención en el sujeto de formación, como fuente inspiradora de la estructura organizativa y la dinámica de la universidad, implica poner en cuestión los límites de los regímenes de verdad establecidos al margen de su voz; reta a descubrir sus sueños, sus deseos, sus necesidades y su pasión antes que forzarlo a adaptarse a un ethos que permanece inamovible frente a su llegada; requiere disponer de otra manera las relaciones entre los actores educativos para que sean reconocidos como interlocutores válidos, que merecen respeto en tanto seres sensibles y racionales con capacidad de autolegislación, porque "una escuela del sujeto es la que refuerza la capacidad de cada cual de convertirse en un actor autónomo, mediante el respeto a la diversidad cultural y, a la vez, mediante el acceso de todos a los conocimientos científicos y técnicos" (TOURAINE, 2002, p. 11) para convertirlos en sabiduría de vida y "construir conjuntamente con los mayores una universidad más a la medida de las nuevas generaciones, o sea, que considere sus sueños, retos, gustos y sus formas de entender la vida" (Tatiana, 18 años) porque, tal como se percibe hoy, "la universidad cierra toda posibilidad real de estar naciendo con los aportes de nosotros los jóvenes, para mí sigue envejecida así uno aprenda cosas nuevas, pero es ella, ella misma como institución" (Daniela, 19 años).

Concebir al sujeto estudiante como vértice de la dinámica universitaria y, junto a ello, aceptar que "la esencia de la educación es la natalidad, el hecho de que en el mundo hayan nacido seres humanos" (ARENDT, 1996, p. 186), es contradecir el pensamiento mercantilista que ha arrasado de manera avasalladora con la confianza en la educación como oportunidad de vida y renovación, y ha configurado como representación social el "no hay nada que hacer" frente a la organización económica mundializada, argumentando que el sistema económico internacional está soportado en una lógica irresistible, que se ha naturalizado y, además, se refuerza de manera constante mediante expresiones que se han cotidianizado como: "quien no marcha al ritmo y de acuerdo a las condiciones de este mundo globalizado se pierde en el círculo de su propio sin sentido, somos demasiado pequeños e impotentes frente al monstruo neoliberal".

La imaginación y las voces jóvenes de los recién llegados que se despliegan en este ejercicio investigativo dejan entrever un anhelo de romper la rutina, desconcentrar el poder, estar presentes no en pequeños fragmentos de la vida institucional, 
sino en toda la realidad para transformarse en ella mientras aportan a la transformación de ésta. Su interés se inclina a romper la continuidad del siempre se ha hecho esto y del mismo modo, a formular lo que no está, a ver lo que no existe y a trasgredir lo socialmente instituido cuando se convierte en barrera para sus agenciamientos, en su doble condición de sujetos y como miembros de un colectivo estudiantil.

Textos como "es un sí se puede rotundo apoyado en la vitalidad de muchos jóvenes que como yo queremos otra cosa, otra educación, otras relaciones, otro país" (Valentina, 19 años) dan cuenta del deseo que tienen los estudiantes de desviar el rumbo, soportado en el cálculo financiero, que han tomado los procesos sociales, entre ellos la educación; según ellos, es un volver al sujeto joven no para magnificarlo en sí mismo, sino para lograr a través de su acción y su palabra comprometida "dar cada vez más y mejor cuenta de fenómenos que se viven como profundamente íntimos, subjetivos, existenciales y en los cuales, empero, reposa cada vez más una parte creciente de nuestra comprensión de la vida social" (MARTUCCELLI, 2007, p. 13), que bien serviría de punto de anclaje para comprender el mundo absorbido por el imperio del dinero, el poder y la apariencia, y buscar formas más éticas de tramitar las grandes contradicciones que de ello se desprenden.

Con apoyo en la referencia a la reflexividad epistémica del sociólogo, que hace Bourdieu (1977, 2001), se puede clarificar que no se trata del retorno intimista, ingenuo y complaciente sobre la persona privada del estudiante. Con este proceso de reflexividad se trata de objetivar, en primera instancia, el lugar que el propio estudiante entra a ocupar en el campo académico y sus posibilidades reales de debatir el discurso hegemónico y hacer ruptura con prácticas pedagógicas diseñadas para perfilarlo en consonancia con el tipo de profesional determinado por el imperio de la productividad que enriquece económicamente. En segunda instancia, y por extensión, importa la posición del sujeto en el campo del poder en el ethos universitario o, dicho de otro modo, qué pasa con su palabra nueva dentro de un discurso que permanece estático y cerrado, cómo son realizadas sus necesidades y qué tanto alcanzan a desarrollarse sus expectativas si ni siquiera es claro el interés de conocerlas, como los mismos estudiantes lo anotan: "hay un potencial dormido, algo así como una gran riqueza humana que pasa desapercibida por el afán de instruir, prescribir y aparecer en el ranking de las mejores universidades académicamente" (Valentina, 20 años).

Desde esta perspectiva y teniendo en cuenta que "el mundo se torna en mundo de la vida, en tanto es materia - sí -, pero lo es de la experiencia humana" (VARGAS, 2007, p. 31), la llegada de estudiantes nuevos a la universidad no se puede asumir como un simple episodio que se planifica y desarrolla con la intención de dar a conocer lo que ya existe y dejar claras las reglas del juego para garantizar su continuidad, porque "la experiencia no tiene que ver con el tiempo lineal de la planificación, de 
la previsión, de la predicción, de la prescripción, sino con el tiempo de la apertura" (SKLIAR; LARROSA, 2009, p. 33). Llegar es sinónimo de renovación, crítica, cambio y posibilidad. Llegar significa acogida a "alguien a quien se reconoce y se aprecia en su diferencia y desde ahí, se le otorgan garantías por su vida, por sus ideas, por sus particularidades" (VARGAS, 2007, p. 36), y para que esto suceda los espacios, los lugares, el discurso y las prácticas tienen que sufrir alteraciones, como consecuencia de la acción crítica de quienes llegan y de su relación con quienes los acogen, “de ahí que nos soñemos tener espacios académicos para producir y hacer resonar palabras nuevas e iniciativas sugestivas que han estado escondidas por el afán de hacer la tarea señalada" (Nicolás, 18 años).

Reconocer en el recién llegado potencial de cambio y renovación no sólo para sí mismo, en cuanto al enfoque y direccionamiento que le puede dar a su proceso formativo y a su vida en general, sino también en cuanto a los aportes que subyacen en su discurso y su acción para la re-fundamentación de la dinámica institucional, implica una mirada autocrítica y abierta de la universidad, capaz de comprender lo inoficioso de perpetuar ideas fundacionales que la siguen ubicando como instancia reproductora de cultura y garante de un status quo, en una sociedad en la que cada vez se hacen más evidentes las promesas incumplidas del pensamiento moderno que la vio nacer.

De acuerdo con lo anterior, hacerse sujeto o nacer, como diría Arendt, en un mundo universitario institucionalizado "en el que las críticas radicales y progresistas de la escuela han sido reducidas a susurros, siendo reemplazadas por la retórica de los expertos del costo-eficiencia" (GIROUX, 2008, p. 67), implica, además de osadía y riesgo, la creación de oportunidades reales para el desarrollo de un compromiso vivo de imaginación, creatividad, razonamiento, sensibilidad y acción crítica, capaz de develar que "todo el truco de la razón pedagógica reside precisamente en la manera como ésta enfatiza lo esencial mientras que aparentemente demanda lo insignificante: obtener respeto por las formas y modos de respeto que constituyen las más visibles y al mismo tiempo más ocultas manifestaciones del orden establecido" (BOURDIEU, 1977, p. 146), tal como anotan los estudiantes en sus recurrentes referencias a la necesidad de enfrentar "la reproducción de males sociales como la corrupción, el caciquismo, la discriminación y el autoritarismo en la cotidianidad universitaria" (Felipe, 21 años).

Precisamente, "la tarea de la educación en una época de desasosiego es la formación de una razón que no crea que educar sea transmitir el sentido de la vida, porque $e l$ sentido no puede darse, sino la formación de una razón imaginativa, que sea capaz de narrar sentidos, de inventar sentidos, en plural" (MÉLICH, 2003, p. 79) o, dicho de otra manera, una razón imaginativa que posibilite la expansión de los sujetos en su diversidad que, en última instancia, es el principio y la finalidad de los 
procesos educativos. Los y las estudiantes abogan por espacios para descubrir/se, inventar/se y producir/se en simultáneo a la apropiación y construcción de los conocimientos incluidos en el perfil profesional. Para ellos y ellas la universidad, además de formar buenos trabajadores, tiene la responsabilidad social de contribuir a la formación de sujetos capaces de provocar/se nuevos nacimientos. Sus textos así lo confirman: "hay mucho joven envejecido, por eso, creo que la universidad tiene que dar herramientas para rejuvenecer el pensamiento o quizás volver a nacer porque un profesional viejo construirá un país con poco futuro" (Nicolás, 18 años).

\section{Reflexión final}

En medio de todos los cambios que trae consigo el primer año de vida universitaria, más que preguntar por la capacidad adaptativa o de acomodación del sujeto, que refuerza los egos institucionales, cabe preguntar por el lugar y la posibilidad de la experiencia del sujeto, es decir, por la visibilización y posicionamiento de su voz en las nuevas relaciones sociales y culturales que se empiezan a tejer; por el descubrimiento de sus potencialidades, posibilidades y opacidades dentro del tumultuoso mundo de la competencia que caracteriza a la academia; por los sentimientos y las emociones que subyacen al éxito o al fracaso académico, por la sensibilidad que queda expuesta en cada tramitación de intereses individuales y colectivos, por las nuevas formas de reivindicar los derechos en muchas ocasiones conculcados $\mathrm{y}$, sobre todo, por la capacidad que ha desarrollado cada sujeto de ex/ponerse en este nuevo contexto de interacción.

Para todos el inicio de la vida universitaria representa cambios e incertidumbres, y necesariamente expone a la tramitación de tensiones entre la permanencia de lo conocido y el advenimiento de lo desconocido, entre la nostalgia, o para muchos alegría, de abandonar un camino que han recorrido por años y la expectativa de transitar nuevos senderos, así como entre la proximidad del inicio y la lejanía de la titulación. Todo empieza cuando se elige una profesión y se inscribe en ella. Ahí el individuo tiene que decidir qué estudiar y dónde estudiar, preguntas que traen implícitas otras consideraciones como escoger un campo disciplinar y junto a ello pensar en la opción de desempeño laboral y en la vida profesional. Así mismo, escoger universidad implica seleccionar un estilo de vida porque cada institución tiene un entorno sociocultural históricamente constituido, ha optado por una forma particular de relacionamiento y de desarrollar sus procesos, además, tiene rituales propios y prácticas establecidas. En el momento de iniciar su vida universitaria cada sujeto empieza a hacer parte de ese ethos, o dicho de otra manera, ha sido "acogido por una familia extensa que hará la función de introducirlo en un mundo, el suyo y el de los otros" (MÈLICH, 2002, p. 35). 
El tema de discusión no es en sí mismo la universidad que se elija o el tipo de profesión por la que se opte, sino las condiciones reales que en ellas tienen los estudiantes recién llegados para superar la sobre estimulación que caracteriza al mundo contemporáneo y tener las experiencias a las que se refiere Skliar y Larrosa (2009), que les permitan cancelar la brecha entre lo que saben y lo que son, entre lo que pasa (y que pueden conocer) y lo que les pasa (como algo a lo que deben atribuir un sentido en relación con ellos mismos).

En situaciones como las actuales, en las que es común encontrar que la vida universitaria, "en su conjunto, se vuelve verdaderamente una abstracción, se ha vuelto autónoma de su sustrato, es decir, de una realidad que no tiene nada de perfecta, sino que es una mezcla compleja de ideal y de bajeza, de generosidades y mezquindades, de grandezas intelectuales y de oscurantismos exacerbados" (MAFFESOLI, 2004, p. 107), recurrir a la experiencia representa posibilidad de atribuirle al conocimiento y a la dinámica universitaria connotaciones diferentes a las de externalidad, mercancía y mera utilidad.

Concebir el inicio de la formación profesional como experiencia es apostarle a otra manera de vivir la universidad y de educar/se en ella; es reconocer en el conocimiento su potencial capaz de con-mover en su intimidad a quienes lo producen, transmiten y/o apropian; es decirle sí a una ciencia que transforma y emancipa; es iniciar de manera consciente el interminable camino de hacerse sujeto desde el reconocimiento de que "siempre nos transformamos desde aquello que ya somos, desde aquello que tenemos" (MÈLICH, 2002, p. 38).

En consecuencia con lo anterior, así como para Arendt ser ciudadano es "una manera pública de ser y estar en el mundo" (BÁRCENA; MÈLICH, 2000, p. 51), se podría decir que ser universitario es una forma de ser y constituir la universidad al unísono que se desenvuelve la vida, se expande la libertad y se configura la subjetividad desde procesos claros de auto-cuestionamiento, o dicho de otro modo: es experienciar la universidad como un acontecimiento impregnado de sensibilidad, vida, incertidumbre, riesgo, inquietud, iniciativa, apuesta... y posibilidad; es, en efecto, haber reconocido y tener claras las normas a las que se acoge el propio ser, esas que tienen el poder de establecerlo, configurarlo o, incluso, desestablecerlo o desconfigurarlo como un sujeto reconocible en un contexto social.

\section{Referencias}

ARENDT, H. La crisis en la educación. In: ARENDT, H. Entre el pasado y el futuro. Barcelona: Península, 1996. p. 269-301.

BENHAABIB, Z. El ser y el otro en la ética contemporánea: femenismo, comunitarismo y posmodernismo. Barcelona: Gedisa, 2006. 
BÁRCENA, F. El delirio de las palabras: ensayo para una poética del comienzo. Barcelona: Herder, 2004.

BÁRCENA, F. El aprendiz eterno: filosofía, educación y arte de vivir. Madrid: Miño y Dávila, 2012.

BÁRCENA. F. Educación y experiencia en el aprendizaje de lo nuevo. Revista Española de Pedagogía, Madrid, n. 223, p. 501-520, sep.-dic. 2002.

BÁRCENA, F.; MÉLICH, J. La educación como acontecimiento ético. Barcelona: Paidós, 2000.

BOURDIEU, P. La reproducción: elementos para una teoría del sistema de enseñanza. Barcelona: Laia, 1977.

BOURDIEU, P. Capital cultural, escuela y espacio social. México, DF: Siglo XXI, 2001.

COFFEY, A.; ATKINSON, P. Encontrar el sentido a los datos cualitativos: estrategias complementarias de investigación. Medellín: Universidad de Antioquia, 2003.

CUBIDES, H. Formación del sujeto político: escuela, medios y nuevas tecnologías de la comunicación y la información. In: LAVERDE, M.C.; DAZA, G.; ZULETA, M. Debates sobre el sujeto: perspectivas contemporáneas. Bogotá: Siglo del Hombre, 2004. p. 37-62.

GADAMER, H. Verdad y método I. Salamanca: Sígueme, 2005.

GIROUX, H. Teoría y resistencia en educación: una pedagogía para la oposición. México, DF: Siglo XXI, 2008.

LARROSA, J. La experiencia de la lectura: estudios sobre literatura y formación. 3. ed. México, DF: Fondo de Cultura Económica, 2004.

MAFFESOLI, M. La transfiguración de lo político: la tribalización del mundo posmoderno. México, DF: Herder, 2004.

MARTUCCELLI, D. Cambio de rumbo: la sociedad a escala del individuo. Santiago de Chile: LOM, 2007.

MÈLICH, J. Filosofía de la finitud. Barcelona: Herder, 2002.

MÈLICH, J. La sabiduría de lo incierto: sobre ética y educación desde un punto de vista literario. Barcelona: Universidad Autónoma, 2003.

NIETZSCHE, F. Así habló Zaratustra. Edición de José Rafael Hernández Arias. Madrid: Valdemar, 2005.

SCHMID, W. En busca de un nuevo arte de vivir. Valencia: Pre-Textos, 2002. 
SIMPSON, O. Cuestión de actitud. Bogotá: Ministerio de Educación Nacional, 2010.

SKLIAR, C.; LARROSA, J. Experiencia y alteridad en educación. Santa Fe: Homo Sapiens, 2009.

TOURAINE, A. A la búsqueda de sí mismo: diálogo sobre el sujeto. Barcelona: Paidós, 2002.

VARGAS, G. Constitución del sujeto y constitución subjetiva de mundo. In: Formación y subjetividad. Bogotá: Universidad Pedagógica Nacional, 2007. p. 29-42.

Recebido em 6 de outubro de 2012.

Aprovado em 28 de novembro de 2013. 